\title{
Comparison of three methods to stabilize bronchoalveolar lavage cells for flowcytometric analysis
}

\author{
Harrie H. M. Eidhof ${ }^{1}$ () | Jan W. Gratama ${ }^{2} \quad$ A. H. Leontine Mulder ${ }^{1,3}$
}

${ }^{1}$ Department of Clinical Chemistry and Laboratory Medicine, Medlon BV, Enschede, The Netherlands

${ }^{2}$ Department of Medical Oncology, Erasmus MC, Rotterdam, The Netherlands

${ }^{3}$ Ziekenhuis Groep Twente, Almelo, The Netherlands

Correspondence

A. H. Leontine Mulder, Medlon BV, Koningsplein 1, 7512KZ Enschede, The Netherlands.

Email: I.mulder@medlon.nl

\begin{abstract}
Background: Flowcytometric analysis of lymphocytes and their subpopulations in bronchoalveolar lavages (BAL) can support the diagnosis of interstitial lung diseases. This analysis should be done within $4 \mathrm{hr}$ after lavage due to rapid cell deterioration. We tested three methods in order to stabilize for at least 28 days the BAL cell populations to allow delayed flowcytometric analysis in order to facilitate external quality assurance (EQA).

Methods: We compared an in-house, dual-step stabilization method for BAL cells with results of two different commercial available stabilization reagents: TransFix ${ }^{\circledR}$ and Streck Cell Preservative ${ }^{\mathrm{TM}}$. All three methods were compared with native BAL cells as reference. BAL samples from six patients were tested on six occasions following stabilization from 1 to 28 days by flow cytometry.

Results: Following stabilization and storage at $4^{\circ} \mathrm{C}$, BAL cell suspensions had stable light scatter patterns and lymphocyte subsets. As expected, rapid deterioration of cells was seen with native BAL cells. The stabilized lavages showed more stable counts of WBC and lymphocyte populations with only minor differences found between the three methods.

Conclusions: If analysis of the BAL cells is performed more than $24 \mathrm{hr}$ after the lavage, stabilized BAL cells are superior to native cells. The in-house method can be used for EQA purposes with stability for at least 28 days. The TransFix and Streck methods might be useful for postponed diagnostic analysis of lavage cells but did not meet our 28 days criterion defined needed for EQA purposes.
\end{abstract}

\section{KEYWORDS}

bronchoalveolar lavage, external quality survey, lymphocyte subsets, stabilization

\section{1 | INTRODUCTION}

Bronchoalveolar lavage (BAL) is performed as a diagnostic procedure on patients with interstitial lung disease. The right middle lobe of the patient's lungs is washed with isotonic saline solution and the aspirated solution analyzed for cell concentration, morphology, differentiation of cells and immunophenotype of lymphocytes. Results may add to diagnosis, especially when suspected for sarcoidosis, extrinsic allergic alveolitis, or idiopathic pulmonary fibrosis (BAL Cooperative Group
Steering Committee, 1990; Barry, Condez, Johnson, \& Janossy, 2002; Lannuzzi, Rybicki, \& Teirstein, 2007; Meyer et al., 2012; Welker, Jörres, Costabel, \& Magnussen, 2004). Since the cells in the lavage fluid are prone to rapid deterioration, the BAL fluid is immediately kept on ice and must be analyzed within $4 \mathrm{hr}$ after lavage (BAL Cooperative Group Steering Committee, 1990; Klech \& Hutter, 1990). The short time required between obtaining the fluid and performing the analysis (Meyer et al., 2012) can be a problem if flowcytometric analysis cannot be done at the site the lavage is being performed. 
BAL cell analysis with immunophenotyping of lymphocytes is currently (2020) done routinely in the Netherlands and Belgium, by more than 40 laboratories. Our laboratory recognized the need for an external quality assurance (EQA) survey, in order to know the interlaboratory variation. However, the deterioration of lavage cells hampers the organization and execution of an EQA program. In order to set up a quality survey with BAL cells we developed a method to stabilize the BAL cells. Our goal was to stabilize the cells in such a way, that the original light scatter and immunophenotypic characteristics of cells in BAL are preserved for a minimum of 28 days and the obtained results remain similar to those of fresh cells. A period of 28 days gives EQA participants sufficient time to analyze the samples and interpret the results, with the opportunity to request a repeat sample if needed. In this article, we present and discuss the comparison of native lavage cells versus stabilized cells using our in-house method and two commercially available fixation reagents, that is, TransFix and Streck Cell Preservative (Streck CP).

\section{2 | MATERIALS AND METHODS}

\section{1 | Specimens}

BAL cells were used from lavages from six different patients that were submitted to our hospital laboratory for analysis and contained more than $10 \times 10^{6}$ nucleated cells. Cell concentrations ranged from 0.150 to $0.825 \times 10^{9} / \mathrm{L}$ and protein concentrations from 0.131 to $0.568 \mathrm{~g} / \mathrm{L}$. After sampling, the lavages were kept on ice. Stabilizations were done within $4 \mathrm{hr}$. All samples were anonymized at the beginning of the study.

\subsection{Stabilization of BAL cells}

Five milliliters of native filtered (through loose gauze) BAL material was stored at $2-8^{\circ} \mathrm{C}$ in a polystyrene tube and used as native $\mathrm{BAL}$ material during 28 days. For our in-house method, we collected cells from $10 \mathrm{ml}$ filtered BAL fluid by centrifugation $10 \mathrm{~min}$ at $400 \mathrm{~g}$ at $2-8^{\circ} \mathrm{C}$. The cell pellet was suspended in Gibco RPMI 1640 medium (Thermo Fisher Scientific, Breda, the Netherlands) with 5\% fetal calf serum (Gibco) and diluted to a cell concentration of $5 \times 10^{9} / \mathrm{L}$. Stabilization was done by adding an equal volume of fixation reagent ( $1 \%$ formaldehyde (10\%, ultrapure EM grade, Polysciences, Inc., Warrington, PA) in phosphate buffered saline (PBS) (Gibco) and incubation at room temperature (RT) for $2 \mathrm{hr}$ with gentle mixing. After a centrifugation step ( $5 \mathrm{~min}$ at $400 \mathrm{~g}, \mathrm{RT}$ ), the cell pellet was suspended in storage solution (3.34\% bovine serum albumin [BSA $22 \%$, Ortho Clinical Diagnostics, Beerse, Belgium] in PBS) at a cell concentration of $5 \times 10^{9} / \mathrm{L}$. This cell suspension was stored at $2-8^{\circ} \mathrm{C}$. Streck Cell Preservative tubes (Streck, Omaha, NE) were used according to the instructions of the manufacturer. TransFix $® / E D T A$ cerebrospinal fluid (CSF) sample storage tubes (Cytomark, Buckingham, UK) were used with a modification for the TransFix procedure (i.e., $5 \mathrm{ml}$ of filtered native BAL material to $500 \mu$ TransFix [a kind gift of Cytomark] instead of the $200 \mu$ l as per the CSF protocol).

\section{3 | Flow cytometry}

On Days 1, 3, 7, 14, 21, and 28, we tested the patient samples either native or stabilized with the in-house method, TransFix, and Streck CP techniques by flowcytometric analysis. Briefly, we took $500 \mu$ l samples of each of the well-mixed tubes (TransFix, Streck CP, or native) for centrifugation at room temperature (5 $\mathrm{min}$ at $400 \mathrm{~g}$ ). Cell pellets were suspended in $50 \mu \mathrm{l}$ of PBS supplemented with $0.5 \%$ BSA. From the inhouse tube, $50 \mu \mathrm{l}$ was used directly. To these cell suspensions $30 \mu \mathrm{l}$ BAL monoclonal mix was added (Table 1), vortexed and incubated for $20 \mathrm{~min}$ at RT in the dark. After Ammonium Chloride lysis $(2 \mathrm{ml})$ and centrifugation, the cell pellet was suspended in $800 \mu \mathrm{l}$ IsoFlow ${ }^{\mathrm{TM}}$ Sheath Fluid (Beckman Coulter, Woerden, The Netherlands). The cells were analyzed on a Navios flow cytometer (Beckman Coulter) within 30 min. Analysis of WBC was done by pipetting $50 \mu$ of in-house cell suspension or $200 \mu$ l of suspensions from TransFix, Streck CP, native cells to $5 \mu \mathrm{l} \mathrm{CD45KO} \mathrm{monoclonal} \mathrm{antibody} \mathrm{(Beckman} \mathrm{Coulter).} \mathrm{After} 20$ min incubation in the dark, $500 \mu$ I IsoFlow Sheath Fluid and $50 \mu$ of Flow Count Fluorosphere beads (Beckman Coulter) were added, mixed, and analyzed. Flowcytometric data analysis was performed with Kaluza software (Beckman Coulter). All lymphocyte subsets were calculated on CD45/SSC gated lymphocytes.

Recovery of WBC, \% lymphocytes or lymphocyte subsets was calculated relative to Day 0 , with the exception of the recovery of the

\begin{tabular}{|llll|}
\hline Antigen & Fluorochrome & Clone & Manufacturer \\
\hline CD3 & APC-Alexa Fluor 750 & UCHT1 & Beckman Coulter \\
\hline CD4 & FITC & $13 B 8.2$ & Beckman Coulter \\
\hline CD8 & APC & B9.11 & Beckman Coulter \\
\hline CD16 & PC7 & $3 G 8$ & Beckman Coulter \\
\hline CD56 & PC7 & N901 (NKH-1) & Beckman Coulter \\
\hline CD45 & KO & B36294 2014-02-19 GB & Beckman Coulter \\
\hline CD19 & HV450 & HIB19 & BD Biosciences \\
\hline CD103 & PE & $7 / 201023-5646-01$ & BD Biosciences \\
\hline
\end{tabular}

TABLE 1 Monoclonal antibodies used 
WBC fixed with the in-house method, this was calculated relative to Day 1.

\section{4 | Statistical analysis}

Statistical analysis was done with SPSS software using repeated measures ANOVA (Dawson \& Trapp, 2001).

\section{3 | RESULTS}

Analysis of the flowcytometric data showed that for all markers studied, a good separation of negative from positive could be obtained regardless of the stabilization method used. Examples of the CD45+ side scatter plots of native and stabilized lavages at Day 7 are shown in Figure 1. Mean results of the six BAL samples at the studied time intervals are shown in Figure 2 for all 10 parameters of interest. The changes in time between the groups were significant for all parameters except the T lymphocytes (defined as CD3+) and \%CD103+ within CD4+ lymphocytes. This significant change was mainly due to the deterioration of the native untreated samples. Exclusion of the native group in the statistical analysis resulted in a significant difference between the three stabilized groups for the $\mathrm{T}$ lymphocytes $(p=.05)$ and CD8 lymphocytes $(p=.01)$, in addition to FSC $(p<.01)$ and SSC $(p<.01)$ (Table 2$)$.

To illustrate the stability and range of change for the different methods of cell preservation, the \% WBC recovery between Days
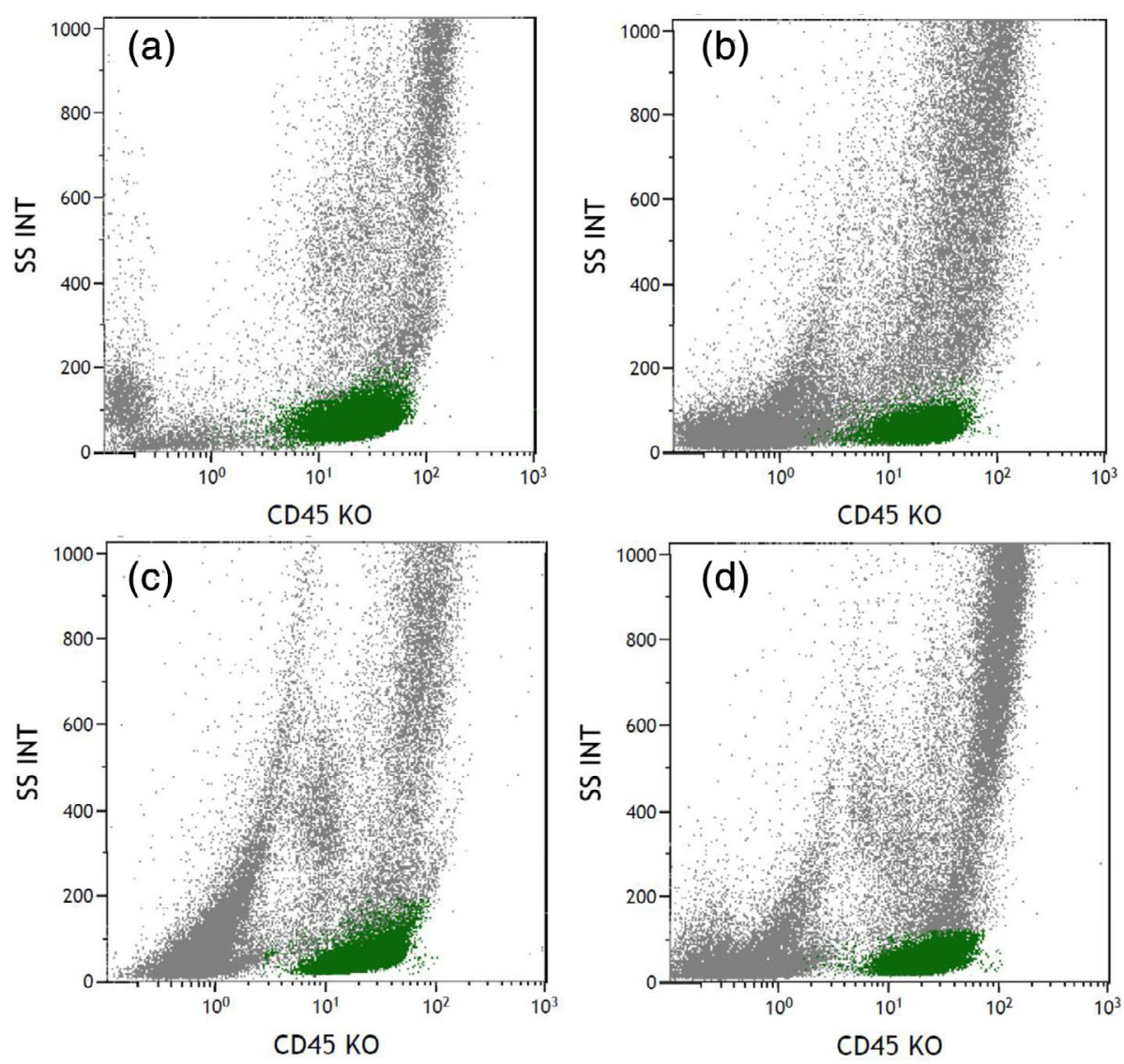

FIGURE 1 CD45+-side scatter plots. (a) Native BAL Day 0, (b) native BAL Day 7, (c) in-house stabilized BAL Day

7, (d) TransFix stabilized BAL Day 7, and (e) Streck CP stabilized BAL Day 7 [Color figure can be viewed at wileyonlinelibrary.com]

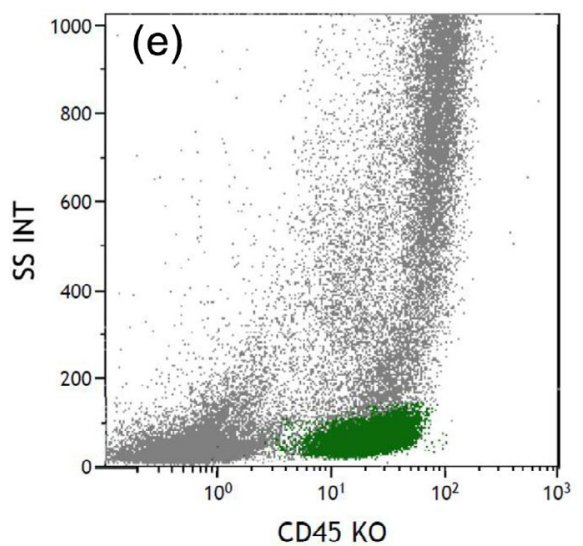


FSC

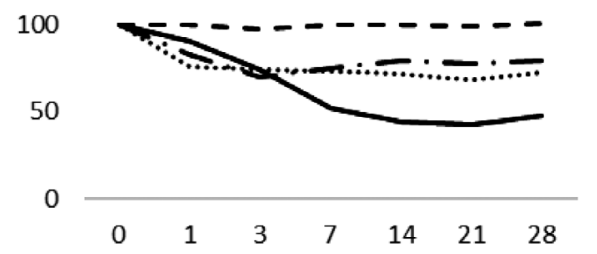

WBC
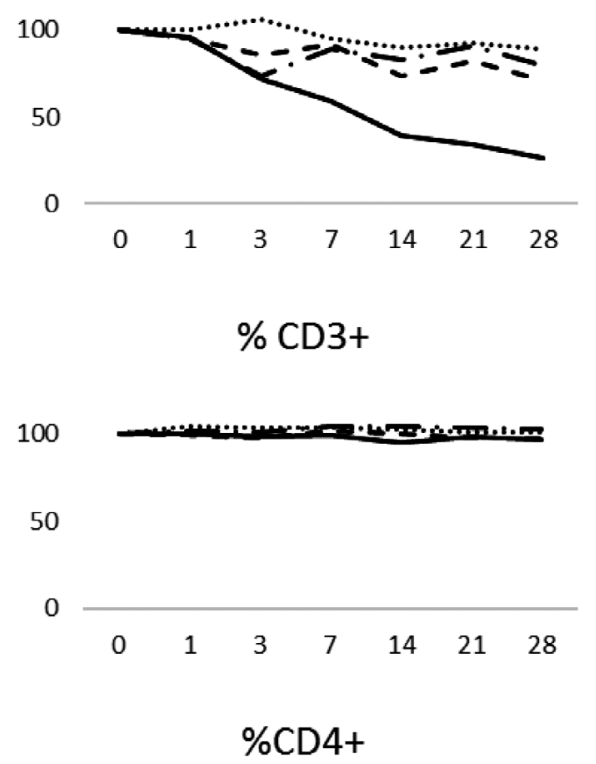

100

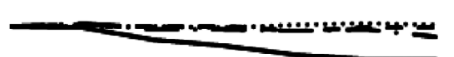

50$$
0
$$

$\begin{array}{lllllll}0 & 1 & 3 & 7 & 14 & 21 & 28\end{array}$

\%CD16+ and/or CD56+

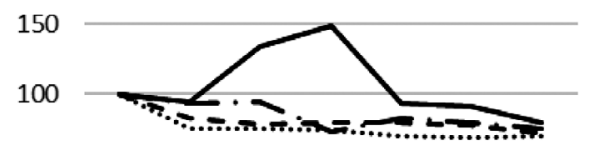

50

0

$\begin{array}{lllllll}0 & 1 & 3 & 7 & 14 & 21 & 28\end{array}$

SSC

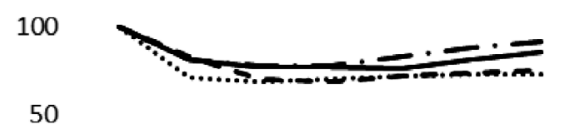

0

$\begin{array}{lllllll}0 & 1 & 3 & 7 & 14 & 21 & 28\end{array}$

\% lymphocytes

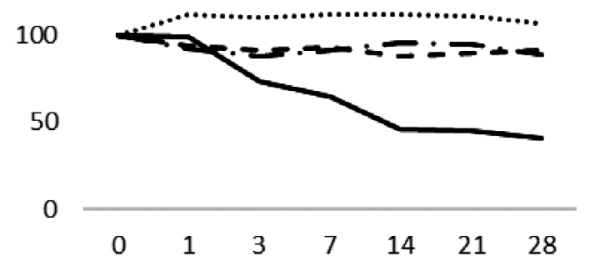

\%CD19+

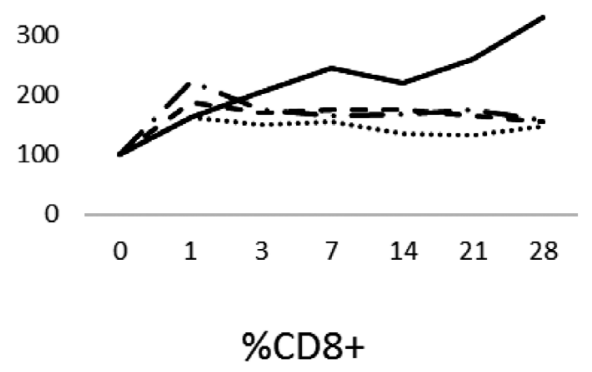

100

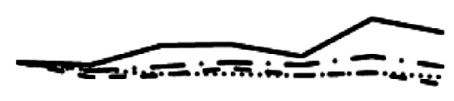

50

0 $\begin{array}{lllllll}0 & 1 & 3 & 7 & 14 & 21 & 28\end{array}$

\%CD103+ within CD4+ subset

100

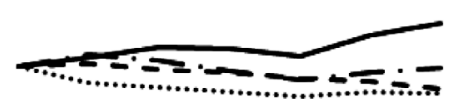

50

0

$\begin{array}{lllllll}0 & 1 & 3 & 7 & 14 & 21 & 28\end{array}$

FIGURE 2 Changes as function of storage time. Given is the recovery (\%) on $Y$-axis at the different time points on the $X$-axis (days) for each stabilization method. (...) In-house; (- - -) TransFix; (- . -) Streck CP; (-) Native 
TABLE 2 Mean recovery at all timepoints

\begin{tabular}{|c|c|c|c|c|c|}
\hline & In-house & TransFix & Streck CP & Native & p stabilized groups \\
\hline WBC & $95(91-100)$ & $85(80-91)$ & $87(76-99)$ & $61(51-71)$ & .21 \\
\hline Lympho & $109(103-115)$ & $92(87-97)$ & $93(87-99)$ & $67(58-76)$ & .06 \\
\hline SSC & $75(72-79)$ & $78(74-81)$ & $86(83-88)$ & $83(80-86)$ & $<.01$ \\
\hline CD3+ cells & $102(100-105)$ & $99(97-101)$ & $103(101-105)$ & $98(95-101)$ & .05 \\
\hline CD3+ CD8+ cells & $94(89-99)$ & $93(90-97)$ & $99(95-102)$ & $110(103-117)$ & .01 \\
\hline CD19+ cells ${ }^{a}$ & $140(115-165)$ & $162(135-189)$ & $166(137-195)$ & $218(172-263)$ & .87 \\
\hline CD3-CD16+,CD56+ cells & $76(70-82)$ & $81(75-88)$ & $85(79-92)$ & $106(91-120)$ & .27 \\
\hline CD103+ within CD4+ & $87(80-94)$ & 95 (91-98) & $99(95-103)$ & $111(103-119)$ & .54 \\
\hline
\end{tabular}

Note: Shown are the mean percentages recovery relative to Day 0 per parameter over all timepoints with $95 \%$ confidence intervals. Mixed measures ANOVA $p$ values are given over the stabilized groups.

${ }^{a}$ Five out of six lavages contained only $1 \%$ CD19+ B cells.

TABLE $3 \%$ WBC recovery relative to Day 0

\begin{tabular}{lllllll} 
& Day 1 & Day 3 & Day 7 & Day 14 & Day 21 & Day 28 \\
\hline In-house & $100^{a}$ & $106(100-113)$ & $95(88-102)$ & $90(73-106)$ & $93(84-101)$ & $89(81-97)$ \\
\hline TransFix & $95(87-102)$ & $85(71-100)$ & $91(79-104)$ & $74(60-88)$ & $82(66-99)$ & $71(54-88)$ \\
\hline Streck CP & $95(59-131)$ & $74(46-102)$ & $89(42-136)$ & $83(52-114)$ & $91(55-126)$ & $79(49-110)$ \\
\hline Native & $96(64-127)$ & $72(53-91)$ & $59(41-78)$ & $39(29-49)$ & $34(24-45)$ & $26(14-38)$ \\
\hline
\end{tabular}

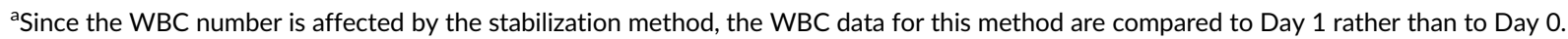

TABLE $4 \quad C V(\%)$ of results of staining after stabilization $(n=6)$

\begin{tabular}{llll} 
Parameter & In-house & TransFix & Streck CP \\
WBC & 5.4 & 7.8 & 6.8 \\
\hline \% lymphocytes & 1.2 & 2.9 & 5.1 \\
FSC & 1.3 & 1.6 & 4.8 \\
SSC & 2.8 & 4.8 & 3.4 \\
\hline \% CD3+ cells & 0.3 & 0.4 & 0.6 \\
\%CD3+ CD4+ & 0.3 & 0.2 & 0.6 \\
\hline \%CD3+ CD8+ & 2.6 & 4.2 & 3.8 \\
\hline \%CD103+ within CD4+ & 2.8 & 3.3 & 4.5 \\
\%CD3-CD16+, CD56+ cells & 5.1 & 4.9 & 5.1 \\
\hline \% CD19+ cells & 3.5 & 1.6 & 2.8 \\
\hline
\end{tabular}

Note: Given is the CV\% of six repetitive stainings of stabilized BAL cells.

Villasmil, \& Frampton, 2007) leading to changes in reactivity patterns with monoclonal antibodies. With the limited panel of antibodies we used (Table 1), we encountered no problems but when other antibodies are used, one has to validate if the antigen is still recognized after stabilization.

Our in-house fixation method is based on formaldehyde fixation in buffered conditions containing serum proteins. Formaldehyde was chosen because of its crosslinking rather than dehydrating properties. We chose to compare this method with commercially available fixatives that are known to stabilize cells in fragile conditions as cerebrospinal fluid (CSF) (de Jongste et al., 2014) or fine needle aspirates. To our opinion these body fluids are similar to BAL fluid because of their low protein content.

Native lavage shows a very rapid deterioration of cell numbers making it not suitable for delayed analysis. When comparing only the stabilized samples, a significant difference within the three methods was found for the CD3+ lymphocytes and CD8+ lymphocytes. Although statistically a significant difference was found for the CD3+ lymphocytes, it can be seen from Table 2 that all three methods resulted in an almost 100\% mean conservation of the CD3 + cell population. Recoveries for the stabilized CD3+ lymphocytes at the different time points varied from $97 \%$ to $105 \%$. The TransFix method showed the best conservation and the lowest variance of the FSC. Regarding the CD8+ T cells the Streck CP reagent showed the best conservation with mean recovery closest to $100 \%$ and lowest variance. All stabilization methods influenced the side scatter (SSC), but this did not influence the gating of the leukocyte population. Technical variation of performed staining on stabilized cells as displayed in Table 4 was equal or sometimes slightly worse, but acceptable, compared to variation of the native material, where $\mathrm{CV}$ from the parameters are all $<5 \%$ (data not shown). As can be seen from Figure 1, sometimes recovery reached values $>100 \%$, especially for the CD19+-cell population (all fixatives) and CD16+/CD56+-cell population ( $d 3$ and $d 7$, native). The CD19+-cell population was $<1 \%$ 
in all but one lavage. Calculation of recoveries of such small populations resulted in values $>100 \%$ (Figure 2). The increment in the CD3-CD16+,CD56+ population cannot be explained by inferior gating or contamination of this population since the sum of the CD3+, CD19+ and CD3-CD16+, CD56+ populations equals $100 \%$. Hypothetically the CD3-CD16+CD56+ could be the population that deteriorates more slowly than the other populations under native conditions, resulting in a relative enrichment. CD103 expression was least conserved by the in-house method for which we have no explanation.

The in-house method is more time consuming and may give some risk on loss of cells with extra centrifugation and aspiration steps in the fixation procedure compared to the TransFix and Streck CP method. In addition due to the methodology of the inhouse stabilization the WBC count of the lavage is standardized $\left(5 \times 10^{9} / \mathrm{L}\right)$ which makes this method not suitable for diagnostic evaluation of BAL. We developed the method in need of a stabilization method that made BAL suitable for an external quality survey. The fact that with this method cells can be brought to a desired cell concentration is indeed an attractive characteristic for quality survey purposes.

The original WBC count of lavages conserved with TransFix of Streck CP can be calculated back, making these reagents possibly suitable for stabilization of lavages in order to postpone the diagnostic analysis, In order to evaluate this possible benefit, we arbitrarily defined the following acceptance criteria: less than 15\% change for leukocyte count, less than $10 \%$ change for cell populations $>10 \%$ and less than $20 \%$ change for cell populations $<10 \%$. Applying these, the samples stabilized with TransFix fulfilled these criteria for 7 days, where most but not all samples stabilized with Streck CP did. However, a great variation of the WBC count was seen with some of the Streck CP stabilized lavages as can be seen in Table 3. This may be due to the fact that the samples stabilized with Streck CP are more diluted than with the other stabilization methods, and the original cell counts were low, ranging $0.150-0.825 \times 10^{9} / \mathrm{L}$. This in combination with the small size of this pilot study makes that no statement can be made whether or not the Streck CP reagent can be used to stabilize BAL cells in order to postpone the diagnostic analysis although \% lymphocyte subsets scored well. More samples need to be measured to obtain more solid information. Our study only monitored the stabilization of the lymphocyte populations and not the other leukocytes that can be present in BAL like neutrophils, eosinophils, or mast cells. Since each lavage has to be investigated for epithelial cells, macrophage inclusions, macrophage foam cells and on indication on asbestos particles, malignant cells, and so forth. always cytospins are prepared. Cellular differentiation to obtain the percentage of nonlymphocyte leukocytes is routinely performed on the cytospins. In case of postponed analysis the cytospins can be made directly after obtaining the lavage, air-dried these can be conserved for weeks.

In conclusion, according to literature BAL cells are prone to rapid deterioration and a general advice is to analyze the cells within $4 \mathrm{hr}$ after sampling. Our results indeed show significant changes over time in WBC count, \% lymphocytes, FSC, and \% CD4, CD 8, CD16,56 or CD103 positive lymphocytes. This study was designed to compare our in-house method with commercially available stabilization products and to prove its suitability to obtain stabilized lavage cells for EQA purposes. ISO/IEC 17043:2010 states that samples selected for proficiency testing should be sufficiently stable to ensure that they will not undergo any significant change throughout the conduct of the proficiency testing, including storage and transport conditions. The 28 day stability of the cells with our in-house method meets this criterion, since the proficiency round with participants only in The Netherlands and Belgium is completed within this period. In this pilot study the commercially available reagents did not fulfill this criterion. However, when flowcytometric diagnostic analysis of the BAL cells is not possible within $4 \mathrm{hr}$, Transfix stabilization might be used to analyze up to 7 days after sampling if immediate stabilization is performed and storage takes place at $2-8^{\circ} \mathrm{C}$. As stated above, for the Streck $\mathrm{CP}$ reagent, more samples need to be measured.

\section{ACKNOWLEDGMENTS}

We kindly acknowledge Professor David Barnett, PhD, FRCPath, for critically reviewing the manuscript and suggestions for improvements. We kindly acknowledge Mrs. M. Vermeer, Clinical Epidemiologist, Ziekenhuis Groep Twente for support on statistical analysis.

\section{ORCID}

Harrie H. M. Eidhof (DD https://orcid.org/0000-0002-7880-2615

\section{REFERENCES}

BAL Cooperative Group Steering Committee. (1990). Bronchoalveolar lavage constituent in healthy individuals, idiopathic pulmonary fibrosis, selected comparison groups. The American Review of Respiratory Disease, 41, 169-202.

Barry, S. M., Condez, A., Johnson, M. A., \& Janossy, G. (2002). Determination of bronchoalveolar lavage leukocyte populations by flow cytometry in patients investigated for respiratory disease. Cytometry, 50, 291-297.

Canonico, B., Zamai, L., Burattinib, V., Grangerd, F., Mannelloc, P., Gobbib, C., ... Papa, S. (2004). Evaluation of leukocyte stabilization in TransFix ${ }^{\circledR}$-treated blood samples by flow cytometry and transmission electron microscopy. Journal of Immunological Methods, 295, 67-78.

Dawson, B., \& Trapp, R. G. (2001). Basic and clinical biostatistics (3rd ed., p. 399). New York: McGraw Hill.

de Jongste, A. H., Kraan, J., van den Broek, P. D., Brooimans, R. A., Bromberg, J. E., van Montfort, K. A., ... Gratama, J. W. (2014). Use of TransFix TM cerebrospinal fluid storage tubes prevents cellular loss and enhances flow cytometric detection of malignant hematological cells after 18 hours of storage. Cytometry Part B, 86B, 272-279.

Klech, H., \& Hutter, C. (1990). Clinical guidelines and indications for bronchoalveolar lavage (BAL). Report of the European Society of Pneumology Task Group on BAL. The European Respiratory Journal, 3 , 937-974.

Lannuzzi, M. C., Rybicki, B. A., \& Teirstein, A. S. (2007). Review article sarcoidosis. The New England Journal of Medicine, 357, 2153-2165.

Meyer, K. C., Raghu, G., Baughman, R. P., Brown, K. K., Costabel, U., du Bois, R. M., ... Brent Wood, B. (2012). An Official American Thoracic Society Clinical Practice Guideline: The clinical utility of bronchoalveolar 
lavage cellular analysis in interstitial lung disease. American Journal of Respiratory and Critical Care Medicine, 185, 1004-1014.

Stewart, J. C., Villasmil, M. N., \& Frampton, M. W. (2007). Changes in fluorescence intensity of selected leukocyte surface markers following fixation. Cytometry Part A, 71A, 379-385.

Welker, L., Jörres, R. A., Costabel, U., \& Magnussen, H. (2004). Predictive value of BAL cell differentials in the diagnosis of interstitial lung diseases. The European Respiratory Journal, 24, 1000-1006.
How to cite this article: Eidhof HHM, Gratama JW, Mulder AHL. Comparison of three methods to stabilize bronchoalveolar lavage cells for flowcytometric analysis.

Cytometry. 2020;1-7. https://doi.org/10.1002/cyto.b.21883 